\title{
Frequency Dependence of Shot Noise in Resonant Diodes under Coherent Tunneling
}

\author{
V.Ya. Aleshikin ${ }^{a}$, L. RegGiani ${ }^{b, *}$ AND M. Rosini ${ }^{b}$ \\ ${ }^{a}$ Institute for Physics of Microstructures, Nizhny Novgorod GSP-105 603600, Russia \\ ${ }^{b}$ National Nanotechnology Laboratory of INFM \\ Dipartimento di Ingegneria dell' Innovazione \\ Università di Lecce, via Arnesano s/n, 73100 Lecce, Italy
}

\begin{abstract}
The current spectral density and the Fano factor of a resonant diode are investigated as a function of frequency up to values just below the inverse of the transit time. We consider the case of coherent tunneling for a symmetric double barrier structure at voltages up to the first current peak at $77 \mathrm{~K}$. At high frequencies the Fano factor is found to become suppressed systematically at a value of 0.25 independently of frequency. This suppression below 0.5 is an indication of coherent against sequential tunneling transport.
\end{abstract}

PACS numbers: 72.70.+m, 72.20.-i, 72.30.+q, 73.23.Ad

\section{Introduction}

Shot noise in double barrier resonant diodes (DBRDs) has been recently investigated in terms of a quantum coherent approach [1] by implementing the original works of Refs. [2-4]. The presence of Pauli principle and Coulomb interaction was found responsible for deviations of the spectral density from the full Poissonian value implied by independent particle transport. One interesting conclusion of this investigation has been the prediction of a suppression of the Fano factor $\gamma=S_{I} /(2 q I)$, with $S_{I}$ - the current spectral density, $q$ - the absolute value of the elementary charge responsible of the steady current $I$, below the value of 0.5 as a consequence of coherent against sequential transport model. The above results were obtained for the low (or zero) frequency value of the current spectral density.

*corresponding author; e-mail: lino.reggiani@unile.it 
Here, the spectral density and the Fano factor of a DBRD are investigated as a function of frequency up to the values just below the inverse of the transit time. Surprisingly enough, at high frequencies the Fano factor is found to become suppressed systematically below the value of 0.5 independently of frequency.

\section{Theoretical model}

The theoretical model implements to the frequency dependent case a coherent approach developed for the typical symmetric double well reported in Fig. 1. We denote by $\Gamma=\Gamma_{\mathrm{L}}+\Gamma_{\mathrm{R}}$ the resonant states width and by $E_{\mathrm{r}}$ the energy of

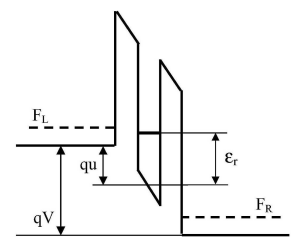

Fig. 1. Sketch of the band profile of the double barrier structure considered here. The bottom of the conduction band in the emitter in the well and in the collector coincides at $V=0$.

the resonant level as measured from the centre of the potential well. Here $\Gamma_{\mathrm{L}, \mathrm{R}}$ are the partial widths due to the tunneling through left and right barrier, respectively. For simplicity, in numerical calculations we consider the case of coherent tunneling when there is only one resonant state with $\Gamma_{\mathrm{L}}=\Gamma_{\mathrm{R}}=\Gamma / 2$ and we take unit square contacts. The kinetic model is developed by assuming that the electron distribution functions in the emitter and in the collector of the DBRT are equilibrium-like, with different electro-chemical potentials $F_{i}$ :

$$
f_{i}\left(\varepsilon, F_{i}\right)=\frac{1}{1+\exp \left(\left(\varepsilon-F_{i}\right) / k_{\mathrm{B}} T\right)},
$$

here $i=\mathrm{L}$ stands for the emitter, $i=\mathrm{R}$ for the collector; $k_{\mathrm{B}}$ is the Boltzmann constant, $T$ - the temperature and $\varepsilon$ - the kinetic carrier energy. The double barrier transparency $D\left(\varepsilon_{z}\right)$ can be written in the following form:

$$
D\left(\varepsilon_{z}\right)=\frac{\Gamma^{2} / 4}{\left(\varepsilon_{z}-\varepsilon_{\mathrm{r}}+q u\right)^{2}+\Gamma^{2} / 4} .
$$

By recalling the expression for the current spectral density at frequency $\omega$ :

$$
S_{I}(\omega)=\int_{-\infty}^{\infty} \mathrm{d} t\langle\delta I(0) \delta I(t)+\delta I(t) \delta I(0)\rangle \exp (\mathrm{i} \omega t),
$$

brackets indicating ensemble average, the current operator $\delta I(t)$ is conveniently written as the sum of two contributions:

$$
\delta I(t)=I(t)-\langle I\rangle=\delta I_{2}(t)+J \delta Q_{\mathrm{QW} 2}(t) .
$$


The first contribution, $\delta I_{2}$, is due to the fluctuations associated with the electrons in the contacts. The second, $J \delta Q_{\mathrm{QW} 2}(t)$, with

$$
J=\frac{1}{C_{\mathrm{L}}+C_{\mathrm{R}}+C_{\mathrm{QW}}} \frac{\partial\langle I\rangle}{\partial u}
$$

is due to the fluctuations associated with the Coulomb interaction of the charge in the quantum well and which are a consequence of the fluctuations of the populations in the contacts. Here $C_{\mathrm{L}, \mathrm{R} . \mathrm{QW}}$ are, respectively, the capacitances of the left barrier, right barrier, and quantum well, and $u$ is the voltage drop between the left barrier and the centre of the quantum well. We note that $J$ is the analogous of a differential dielectric relaxation rate.

In analogy with the low frequency case, $\omega=0$, we find that the spectral density of current fluctuations is the sum of three terms:

$$
S_{I}(\omega)=S_{1}(\omega)+S_{2}(\omega)+S_{3}(\omega),
$$

where

$$
\begin{aligned}
& S_{1}(\omega)=\int_{-\infty}^{\infty} \mathrm{d} t\left\langle\delta I_{2}(0) \delta I_{2}(t)+\delta I_{2}(t) \delta I_{2}(0)\right\rangle \exp (\mathrm{i} \omega t) \\
& S_{2}(\omega)=J \int_{-\infty}^{\infty} \mathrm{d} t\left\langle\delta I_{2}(0) \delta Q_{\mathrm{QW} 2}(t)+\delta I_{2}(t) \delta Q_{\mathrm{QW} 2}(0)+\delta Q_{\mathrm{QW} 2}(0) \delta I_{2}(t)\right. \\
& \left.\quad+\delta Q_{\mathrm{QW} 2}(t) \delta I_{2}(0)\right\rangle \exp (\mathrm{i} \omega t) \\
& S_{3}(\omega)=J^{2} \int_{-\infty}^{\infty} \mathrm{d} t\left\langle\delta Q_{\mathrm{QW} 2}(0) \delta Q_{\mathrm{QW} 2}(t)+\delta Q_{\mathrm{QW} 2}(t) \delta Q_{\mathrm{QW} 2}(0)\right\rangle \exp (\mathrm{i} \omega t) .(
\end{aligned}
$$

According to the Ramo-Shockley theorem, for the model under consideration the current fluctuation in Eq. (4) satisfies the following expression [2]

$$
\delta I=\eta \delta I_{\mathrm{L}}-(1-\eta) \delta I_{\mathrm{R}},
$$

where $\eta$ is the fraction of applied voltage $V$ which drops between the emitter and the centre of the quantum well, $I_{\mathrm{L}}$ the emitter current and $I_{\mathrm{R}}$ the collector current.

By introducing the dimensionless parameter $\lambda$,

$$
\lambda=\frac{\hbar \Gamma}{\Gamma_{\mathrm{L}} \Gamma_{\mathrm{R}}} \frac{1}{\left(C_{\mathrm{L}}+C_{\mathrm{R}}+C_{\mathrm{QW}}\right)} \frac{\partial\langle I\rangle}{\partial u}=\frac{\hbar \Gamma}{\Gamma_{\mathrm{L}} \Gamma_{\mathrm{R}}} J,
$$

whose value, when different from zero, is a measure of the importance of Coulomb interaction, the expressions for $S_{1}, S_{2}, S_{3}$ are found to take the following explicit forms: 


$$
\begin{aligned}
& S_{1}(\omega)=\frac{q^{2}}{2 \pi \hbar} \sum_{p_{\perp}, \pm} \int \mathrm{d} E\left\{D(E) D(E \pm \hbar \omega)\left(1+\eta^{2} \frac{\hbar^{2} \omega^{2}}{\Gamma_{\mathrm{R}}^{2}}\right) f_{\mathrm{L}}\left(E+E_{\perp}\right)\right. \\
& \times\left[1-f_{\mathrm{L}}\left(E \pm \hbar \omega+E_{\perp}\right)\right]+D(E) D(E \pm \hbar \omega)\left[1+(1-\eta)^{2} \frac{\hbar^{2} \omega^{2}}{\Gamma_{\mathrm{L}}^{2}}\right] \\
& \times f_{\mathrm{R}}\left(E+E_{\perp}\right)\left[1-f_{\mathrm{R}}\left(E \pm \hbar \omega+E_{\perp}\right)\right]+[D(E)(1-\eta)+D(E \pm \hbar \omega) \eta \\
& \left.-D(E) D(E \pm \hbar \omega)\left(1+\frac{\eta(1-\eta)(\hbar \omega)^{2}}{\Gamma_{\mathrm{L}} \Gamma_{\mathrm{R}}}\right)\right] f_{\mathrm{L}}\left(E+E_{\perp}\right) \\
& \times\left[1-f_{\mathrm{R}}\left(E \pm \hbar \omega+E_{\perp}\right)\right]+[D(E) \eta+D(E \pm \hbar \omega)(1-\eta)-D(E) D(E \pm \hbar \omega) \\
& \left.\left.\times\left(1+\frac{\eta(1-\eta)(\hbar \omega)^{2}}{\Gamma_{\mathrm{L}} \Gamma_{\mathrm{R}}}\right)\right] f_{\mathrm{R}}\left(E+E_{\perp}\right)\left[1-f_{\mathrm{L}}\left(E \pm \hbar \omega+E_{\perp}\right)\right]\right\}, \\
& S_{2}(\omega)=-\frac{q^{2} \lambda}{2 \pi \hbar} \sum_{p_{\perp}, \pm} \int \mathrm{d} E D(E) D(E \pm \hbar \omega)\left\{2 \frac{\Gamma_{\mathrm{L}}}{\Gamma} f_{\mathrm{L}}\left(E+E_{\perp}\right)\right. \\
& \times\left[1-f_{\mathrm{L}}\left(E \pm \hbar \omega+E_{\perp}\right)\right]-2 \frac{\Gamma_{\mathrm{R}}}{\Gamma} f_{\mathrm{R}}\left(E+E_{\perp}\right)\left[1-f_{\mathrm{R}}\left(E \pm \hbar \omega+E_{\perp}\right)\right] \\
& +\frac{\left(\Gamma_{\mathrm{R}}-\Gamma_{\mathrm{L}}\right)}{\Gamma}\left[f_{\mathrm{L}}\left(E+E_{\perp}\right)\left(1-f_{\mathrm{R}}\left(E \pm \hbar \omega+E_{\perp}\right)\right)\right. \\
& \left.\left.+f_{\mathrm{R}}\left(E+E_{\perp}\right)\left(1-f_{\mathrm{L}}\left(E \pm \hbar \omega+E_{\perp}\right)\right)\right]\right\} \\
& S_{3}(\omega)=\frac{q^{2} \lambda^{2}}{2 \pi \hbar} \sum_{p_{\perp}, \pm} \int \mathrm{d} E D(E) D(E \pm \hbar \omega)\left\{\frac{\Gamma_{\mathrm{L}}^{2}}{\Gamma^{2}} f_{\mathrm{L}}\left(E+E_{\perp}\right)\right. \\
& \times\left[1-f_{\mathrm{L}}\left(E \pm \hbar \omega+E_{\perp}\right)\right]+\frac{\Gamma_{\mathrm{L}} \Gamma_{\mathrm{R}}}{\Gamma^{2}} f_{\mathrm{L}}\left(E+E_{\perp}\right)\left[1-f_{\mathrm{R}}\left(E \pm \hbar \omega+E_{\perp}\right)\right] \\
& +\frac{\Gamma_{\mathrm{L}} \Gamma_{\mathrm{R}}}{\Gamma^{2}} f_{\mathrm{R}}\left(E+E_{\perp}\right)\left[1-f_{\mathrm{L}}\left(E \pm \hbar \omega+E_{\perp}\right)\right] \\
& \left.+\frac{\Gamma_{\mathrm{R}}^{2}}{\Gamma^{2}} f_{\mathrm{R}}\left(E+E_{\perp}\right)\left[1-f_{\mathrm{R}}\left(E \pm \hbar \omega+E_{\perp}\right)\right]\right\},
\end{aligned}
$$

where \pm indicates absorption and emission processes, respectively.

\section{Results and discussion}

Figure 2 reports the current spectral density (Fig. 2a) and the corresponding Fano factor (Fig. 2b) as a function of the applied voltage, here measured in convenient normalised units, at different frequencies for a typical DBRD with the 

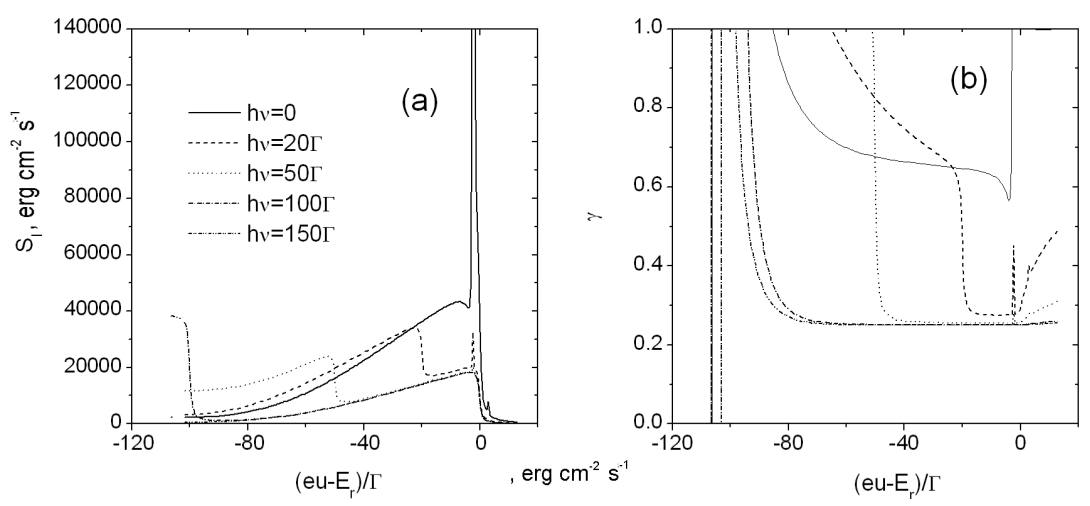

Fig. 2. Dependence of the current spectral density (a) and Fano factor (b) on the applied voltage at different frequencies for the symmetric DBRD at $77 \mathrm{~K}$ with the following parameters: contact concentration $5 \times 10^{17} \mathrm{~cm}^{-3}, \Gamma=0.5 \mathrm{meV}$, length of the right and left barrier $=100 \mathrm{~A}$, energy of the resonant level $=50 \mathrm{meV}$.

parameters given in the figure caption. Focusing our attention to the region of negative voltages (i.e. before the current peak), we have found that at increasing frequency the spectral density exhibits a systematic drop at voltages corresponding to the frequency considered. With respect to the zero frequency curve, the spectral density is enhanced at increasing negative voltages and suppressed at decreasing negative voltages. This reshaping of the spectral density is better monitored by the behaviour of the Fano factor which is reported in Fig. 2b. (In Fig. 2b the spike of the Fano factor around -100 corresponds to the negative current value associated with a small negative value of the external applied voltage.) In particular, let us discuss the interesting high frequency region $\hbar \omega \rightarrow \infty$ (but in any case below the value corresponding to the inverse of the transit time). In this limit, emission processes are no longer possible and only absorption processes remain active. Since for energies $E$ which correspond to the occupied electron states $D(E+\hbar \omega) \rightarrow 0$, we conclude that $S_{2,3}(\omega) \rightarrow 0$. By taking into account that, when $\hbar \omega \rightarrow \infty, D(E+\hbar \omega)(\hbar \omega)^{2}=\Gamma_{\mathrm{L}} \Gamma_{\mathrm{R}}$, in this limit we find

$$
\begin{aligned}
& S_{1}(\infty)=\frac{q^{2}}{2 \pi \hbar} \sum_{p_{\perp}} \int \mathrm{d} E D(E)\left[\eta^{2} \frac{\Gamma_{\mathrm{L}}}{\Gamma_{\mathrm{R}}} f_{\mathrm{L}}\left(E+E_{\perp}\right)+(1-\eta)^{2} \frac{\Gamma_{\mathrm{R}}}{\Gamma_{\mathrm{L}}} f_{\mathrm{R}}\left(E+E_{\perp}\right)\right. \\
& \left.+(1-\eta)^{2} f_{\mathrm{L}}+\eta^{2} f_{\mathrm{R}}\right] .
\end{aligned}
$$

By recalling the definition of the average current:

$$
\langle I\rangle=\frac{q}{2 \pi \hbar} \sum_{p_{\perp}} \int \mathrm{d} E D(E)\left[f_{\mathrm{L}}\left(E+E_{\perp}\right)-f_{\mathrm{R}}\left(E+E_{\perp}\right)\right],
$$

in the high voltage limit $q V \gg k_{\mathrm{B}} T$ it is $f_{\mathrm{L}} \gg f_{\mathrm{R}}$ and for the high frequency Fano factor $\gamma(\infty)$ we find: 


$$
\gamma(\infty)=\frac{1}{2}\left[\eta^{2} \frac{\Gamma_{\mathrm{L}}}{\Gamma_{\mathrm{R}}}+(1-\eta)^{2}\right]=\frac{1}{2} \frac{\Gamma_{\mathrm{L}}}{\Gamma_{\mathrm{L}}+\Gamma_{R}} .
$$

The expression (16) should be compared with that corresponding to the sequential tunneling model which, following Eqs. (35), (69), and (70) of Ref. [5], is given by

$$
\gamma^{\mathrm{seq}}(\infty)=\eta^{2}+(1-\eta)^{2}=\frac{\Gamma_{\mathrm{R}}^{2}+\Gamma_{\mathrm{L}}^{2}}{\left(\Gamma_{\mathrm{R}}+\Gamma_{\mathrm{L}}\right)^{2}} .
$$

We note that, in the coherent tunneling model for a symmetric barrier diode, the high frequency shot noise is suppressed to a minimum value of 0.25 , that is half of the corresponding sequential tunneling value, as shown by the drop of the Fano factor at frequencies corresponding to the voltage drop in Fig. 2b.

\section{Acknowledgments}

Research performed within the project "Noise Models and Measurements in Nanostructures". Partial support by the Italian Ministry of Education and Research, the NATO Collaborative Linkage Grant PST.EAP.CLG 980629, the Italian Ministry of Foreign Affairs through the Volta Landau Center (the fellowship of V.Ya.A.) is gratefully acknowledged. We thank Prof. M. Büttiker of Geneva University for valuable discussions on the subject.

\section{References}

[1] V.Ya. Aleshkin, L. Reggiani, N.V. Alkeev, V.E. Lyubchenko, C.N. Ironside, J.M.L. Figuerido, C.R. Stanley, cond-matter/0304077 (2003), Phys. Rev. B 70, 115321 (2004).

[2] D.V. Averin, J. Appl. Phys. 73, 2593 (1993).

[3] Y.M. Blanter, M. Büttiker, Phys. Rev. B 59, 10217 (1999).

[4] Y. Wei, B. Wang, J. Wang, H. Guo, Phys. Rev. B 60, 16900 (1999).

[5] G. Iannaccone, M. Macucci, B. Pellegrini, Phys. Rev. B 55, 4539 (1997). 